\title{
Desarrollo sostenible en las Mipymes de Ecuador y su impacto en el consumidor
}

\author{
Sustainable development in the MSMEs of Ecuador \\ and its impact on the consumer
}

Sánchez-González Irene Patricia

Universidad Técnica de Machala (Ecuador) isanchez@utmachala.edu.ec

\section{Benítez-Luzuriaga Francisco Vladimir} Universidad Técnica de Machala (Ecuador) fbenitez@utmachala.edu.ec

Moscoso-Parra Ana Elizabeth Universidad Técnica de Machala (Ecuador) elmoscoso@utmachala.edu.ec

Muñoz-Suárez Manuel Universidad Técnica de Machala (Ecuador) mmunoz@utmachala.edu.ec

\section{Revista Cumbres Vol.6 №1}

Versión electrónica ISSN 1390-3365 http://investigacion.utmachala.edu.ec/revistas/index.php/Cumbres 


\title{
RESUMEN
}

El presente trabajo propone un análisis del desarrollo sostenible de las micro, pequeñas y medianas empresas, y su impacto en los consumidores. Partiendo de las percepciones de 267 clientes de las MIPYMES en Ecuador, se constata que tanto el valor percibido (valor social, emocional, por dinero y calidad) como las iniciativas de desarrollo sostenible (económicas, sociales y medioambientales) conllevan a tener un efecto positivo y significativo sobre el comportamiento del consumidor. A través de un muestreo de tipo probabilístico, donde se realizaron encuestas personales a una muestra representativa en las ciudades de Quito, Guayaquil y Machala, se pudo determinar que la sostenibilidad, unida a los cambios constantes en los gustos, preferencias y necesidades del mercado, supone un desafío en las estrategias de marketing que establecen las empresas para generar un mayor impacto en los consumidores, logrando un posicionamiento eficiente.

Palabras clave: Desarrollo sostenible, MIPYMES, comportamiento del consumidor, estrategias de marketing, posicionamiento.

\begin{abstract}
The present work proposes an analysis of the sustainable development of micro, small and medium enterprises, and their impact on consumers. Based on the perceptions of 317 MSMEs clients in Ecuador, it is found that both the perceived value (social, emotional value, for money and quality) and sustainable development initiatives (economic, social and environmental) lead to a positive effect and significant about consumer behavior. Through a probabilistic type sampling, where personal surveys were conducted to a representative sample in the cities of Quito, Guayaquil, Cuenca and Machala, it was possible to determine that sustainability, together with constant changes in the tastes, preferences and needs of the market, is a challenge in marketing strategies that establish companies to generate a greater impact on consumers, achieving an efficient positioning.
\end{abstract}

Keywords: Sustainable development, MSMEs, consumer behavior, marketing strategies, positioning.

\section{Cumbres}




\section{INTRODUCCIÓN}

El concepto de sostenibilidad incluye no sólo la búsqueda de la calidad ambiental, sino también la equidad y la justicia social como criterios y valores que es preciso contemplar en los procesos de formación (Minguet \& Angels Ull Solís, 2009).

El informe de Brundtland del año 1987, de la Comisión del Medio Ambiente y Desarrollo, define al Desarrollo Sostenible (DS) como aquel "que satisface las necesidades de la generación presente, sin comprometer la capacidad de las generaciones futuras de satisfacer sus propias necesidades" (Bermejo, 2014, p.16).

América Latina enfrenta grandes retos de cara a los desafíos globales planteados en la Agenda 2030 para el Desarrollo Sostenible DS, una plataforma ambiciosa y universal que involucra a todos los países (ONU, 2015), y las Negociaciones Comerciales del siglo XXI. Se espera que la Agenda 2030 procure una profunda transformación del desarrollo sostenible, orientada a atender las necesidades de las personas. (Álvarez, 2016).

Respecto a la transformación de las empresas, en América Latina cada vez más están interesas en este DS, las micro, pequeñas y medianas empresas, MIPYMES. Las MIPYMES son importantes para el tejido económico de un país por la cantidad de empleo que generan, porque su producción está vinculada al mercado interno y porque gran parte de la población y de la economía local dependen de su actividad y de los impactos positivos y negativos que producen (Santeli, 2016). Sin embargo, no se encuentra fácilmente disponible la información mínima que permita conocer la situación de estas empresas en cuanto a su definición, al número de estas empresas, la conformación sectorial y su importancia económica (Saavedra García, Moreno Uribe, \& Hernández Callejas, 2008). En Ecuador, según datos del grupo de investigación COMARK, una microempresa posee de 1 a 9 trabajadores; la pequeña empresa de 10 a 49; y, la mediana, de 50 a 199.

En este contexto, investigar sobre el Desarrollo Sostenible de las MIPYMES del Ecuador resulta de interés para avanzar en el conocimiento relativo al impacto o posicionamiento en el consumidor. Por ello, el principal objetivo de este trabajo es analizar la naturaleza y el alcance de las iniciativas del Desarrollo Sostenible de las MIPYMES percibido por el consumidor.

Los consumidores, como parte interesada, juegan un papel muy importante porque esperan evidencias concretas del compromiso social, medioambiental y económico de las MIPYMES (Lavorata, 2014), por lo que resulta conveniente analizar el impacto de su comportamiento.

En este sentido, la propuesta que se presenta pretende disminuir el gap identificado en la literatura en lo referente al desarrollo sostenible de las MIPYMES, y respecto al impacto que ésta genera en el consumidor.

A través de un modelo de relaciones, se analiza el impacto que generan las políticas de desarrollo sostenible en los consumidores, sobre la cadena de efectos capital de marca y lealtad. 


\section{MATERIALES Y MÉTODOS}

\section{Desarrollo sostenible y su impacto en el consumidor}

La sostenibilidad, emerge como tópico de interés a partir de la década de los 80 (Yip y Bocken, 2018). Las empresas se apropiaron del término, asociándolo desde sus inicios con valores positivos (Aggeri et al., 2005), convirtiéndo dichas acciones en una herramienta de marketing, por la imagen que permite transmitir y los beneficios que genera.

La sostenibilidad en el sector empresarial se define como "un enfoque comercial sistemático y una estrategia, que considera el impacto social y ambiental a largo plazo de todas las conductas motivadas económicamente de una empresa, en interés de los consumidores, empleados y propietarios o accionistas" (Bergman et al., 2017, p. 10); Yip y Bocken (2018, p. 151) la definen como "generar beneficios reduciendo significativamente los impactos negativos para el medio ambiente y la sociedad".

Existe una creciente preocupación por la sostenibilidad por parte de los MIPYMES (Gulyás, 2011), incorporándolas en sus prácticas comerciales diarias (Sanclemente-Téllez, 2015). Se observan acciones, como: contribuir a una mejora en la calidad de vida de empleados, proveedores y consumidores (Lombart y Louis, 2014), propulsar empleo digno (Lavorata, 2014), innovación (Reinartz et al., 2011); e, intervenir en causas sociales y medioambientales con la definición de precios de descuento y promociones (Andrews et al., 2014).

He y Hong (2017) concluyen que las MIPYMES que adoptan prácticas sostenibles, se vuelven más competitivas y como consecuencia, mejoran su imagen corporativa y su posición en el mercado, facilitando su crecimiento a largo plazo (Bobe y Dragomir, 2010).

\section{Posicionamiento en el mercado}

El origen del posicionamiento surge a partir de los estudios de marketing mix en los años 40 (Horsky y Nelson, 1992), evolucionando de las discusiones de segmentación de mercado. Sin embargo hoy, la llave del éxito de las MIPYMES está en la implementación eficaz del concepto de marketing a través de estrategias de posicionamiento aplicadas en el mercado (Serralvo y Tadeu, 2005). Se trata de agrupar clientes y competidores que tiene como consecuencia las percepciones, creencias y actitudes de los clientes, relacionadas con la forma en que las personas perciben los mercados (Hooley y Sauders, 1996).

En base a la literatura, se plantean las siguientes hipótesis:

H1: El compromiso de la MIPYME en el desarrollo sostenible tiene un efecto positivo y significativo sobre el posicionamiento en el mercado.

H2: El posicionamiento en el mercado tiene un efecto positivo y significativo sobre el capital de marca. 


\section{Capital de marca}

El capital de marca se propone como un activo que se genera a través de interacciones duraderas y relaciones a largo plazo entre las marcas y sus clientes (Davcik et al., 2015). Para Aaker (1991) el capital de marca es el acumulado de elementos del activo y pasivo, ligados a una marca, a su nombre, o a sus símbolos que le aportan cualquier cosa (más valor o menos valor) a la empresa y a sus clientes.

Con el aumento de los niveles de competencia, la globalización y la creciente conciencia de que las marcas son uno de los intangibles más valiosos de una empresa (Girard et al., 2017), el capital de marca es uno de los constructos más estudiados en el área de marketing sin embargo su aplicación en el ámbito de las MIPYMES es una inquietud relativamente reciente (Shen, 2010; Awan et al., 2017).

En este sentido, algunas investigaciones (Yoo et al., 2000; Yoo y Donthu, 2001) destacan que son los consumidores quienes asocian los "buenos" pensamientos, las imágenes y desarrollan percepciones, así como actitudes positivas, respecto de su marca.

En este contexto, es la empresa la que construye su capital de marca según sus estrategias realizadas, mientras que los consumidores, la evalúan en función de lo que perciben en las acciones comunicacionales y en las características del producto (Stahl et al., 2012). Por lo que, los consumidores pueden esperar ciertas acciones éticas, precio y calidad basados en el valor percibido (Chang y Jai, 2015).

La sostenibilidad y el capital de marca son constructos que se relacionan en el sector de las MIPYMES, el estudio de Cai y He (2014) determina el impacto positivo de la responsabilidad ambiental en el capital de marca, por lo tanto enunciamos la tercera hipótesis:

H3: El compromiso de las MIPYMES en el desarrollo sostenible tiene un efecto positivo y significativo sobre el capital de marca.

\section{Lealtad}

La lealtad, es un proceso no solo un acto (Torres-Moraga et al., 2008), en donde es importante considerar tanto las actitudes, como el comportamiento de compra y recompra (Gallarza et al., 2016), en ese contexto, la lealtad del consumidor hacia el minorista puede ser observada como un componente del capital de marca, porque las actividades mentales y físicas que llevan cabo los individuos resultan en decisiones y acciones para comprar, pagar y usar productos y/o servicios (Sheth et al, 1999).

En el sector de las MIPYMES, el capital de marca ha sido fuertemente vinculado con la lealtad, Su y Chang (2018) concluyen que el capital de marca contribuye a generar la lealtad del consumidor hacia la empresa.

Con todo, las investigaciones relacionadas en el sector, evidencian la relación positiva del capital de marca sobre la lealtad (Cho y Jang, 2017; Su y Chang, 2018). Por ello, en base a la literatura existente, se propone la cuarta hipótesis: 
H4: El capital de marca tiene un efecto positivo y significativo sobre la lealtad.

En el Figura se expone el modelo que representan las relaciones planteadas en forma de hipótesis.

Figura 1. Modelo teórico

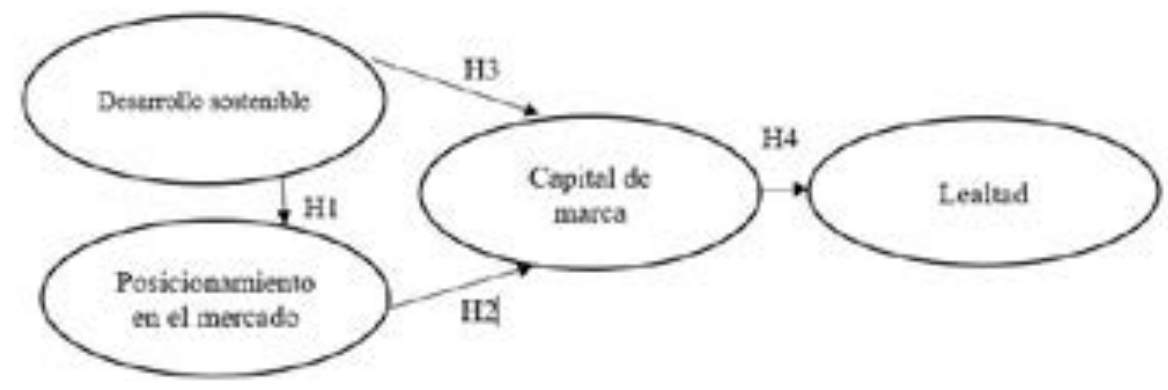

\section{METODOLOGÍA}

\section{Muestra y recogida de información}

Con el fin de recoger la información que ayude a contrastar las hipótesis planteadas, se realizó un cuestionario ad-hoc con preguntas cerradas, donde el consumidor debía indicar su grado de acuerdo o de acuerdo en una escala de Likert, aplicando una entrevista a 267 consumidores, durante los meses de enero a marzo 2018 en Ecuador, en las ciudades de Quito, Guayaquil y Machala, a través de un muestreo probabilístico.

Tabla 1. Datos de la investigación

\begin{tabular}{|l|l|}
\hline UNIVERSO & CONSUMIDORES MAYORES DE 18 AÑOS \\
\hline Ámbito geoFigura & Ecuador: Quito, Guayaquil, Machala \\
\hline Tamaño de la muestra & 267 \\
\hline Diseño muestra & Encuesta personal \\
\hline Técnicas estadísticas & $\begin{array}{l}\text { Análisis descriptivo, Análisis Factorial Ex- } \\
\text { ploratorio, Análisis Factorial Confirmatorio } \\
\text { (AFC), Modelo de Ecuaciones Estructurales }\end{array}$ \\
\hline Programa estadístico & IBM SPSS y Smart PLS 3 \\
\hline
\end{tabular}

\section{Medición de variables}

Los ítems que se han utilizado han sido extraídos y adaptados de diversas escalas utilizadas en la literatura de marketing. Se utilizaron las escalas de medición tipo Likert de cinco puntos, siendo 1 totalmente de desacuerdo y 5 totalmente de acuerdo.

Para la medición del DS, adaptamos la escala de Lavorata (2014), para medir el capital de marca utilizamos la escala de Shen (2010); y, la lealtad fue medida a partir de la escala propuesta y validada en el trabajo de Arnett (2003). 


\section{RESULTADOS}

\section{Análisis de los resultados}

En la primera fase se realizó un Análisis Factorial Exploratorio utilizando el programa SPSS, con el objetivo de estudiar la dimensionalidad de las diferentes variables retenidas.

En la segunda fase se validó el instrumento mediante una Análisis Factorial Confirmatorio y posteriormente se estimó el modelo estructural, utilizando la técnica PLS (Hair, 2016).

A partir de los resultados del Análisis Factorial Confirmatorio, se puede confirmar la fiabilidad de todas las escalas, ya que tanto los valores para la fiabilidad compuesta (CR) como para el coeficiente Alfa de Cronbach (CA) son superiores a los valores recomendados de 0,7 (Nunnally y Bernstein, 1994), o superiores a 0,8 (Carmines y Zeller, 1975), para todos los constructos de primer orden.

Por otra parte, en el contexto de PLS-SEM, se estima el modelo de ecuaciones a través de mínimos cuadrados parciales (PLS), y se comprueba el poder explicativo del modelo estructural a través de coeficientes de determinación:

Tabla 2. Contraste de hipótesis

\begin{tabular}{|l|l|l|l|}
\hline HIPÓTESIS & 10,686 & Aceptada \\
\hline $\begin{array}{l}\text { H1 Desarrollo sostenible } \rightarrow \\
\text { Posicionamiento en el mer- } \\
\text { cado }\end{array}$ & $0,108^{* *}$ & 13,243 & Aceptada \\
\hline $\begin{array}{l}\text { H2 Posicionamiento en el } \\
\text { mercado } \rightarrow \text { Capital de marca }\end{array}$ & $0,615^{* *}$ & 4,567 & Aceptada \\
\hline $\begin{array}{l}\text { H3 Desarrollo sostenible } \rightarrow \\
\text { Capital de marca }\end{array}$ & $0,272^{* *}$ & 33,654 & Aceptada \\
\hline $\begin{array}{l}\text { H4 Capital de marca } \rightarrow \text { Leal- } \\
\text { tad }\end{array}$ & $0,456^{* *}$ & & \multicolumn{2}{l}{ CONTRASTE } \\
\hline
\end{tabular}

Nota: R2 (Capital de marca) $=0,573 ; \mathrm{R} 2($ Lealtad) $=0,649 ; \mathrm{R} 2$ (Valor percibido) $=1,00$; R2 (Desarrollo sostenible $)=1,00$. Q2 $($ Capital de marca $)=0,530 ;$ Q2 $($ Lealtad $)=0,544$; Q2 $($ Valor percibido $)=0,677$; Q2 (Posicionamiento) $=0,578 ; * * p<0.01, * \mathrm{p}<0.05$

Fuente: Elaboración propia

\section{CONCLUSIONES}

La investigación realizada ha permitido disminuir la brecha existente sobre el concepto del Desarrollo sostenible en las MIPYMES, y su relación con el comportamiento del consumidor; lo que facilita el posicionamiento del producto en el mercado.

En primer lugar, se ha analizado la relación del DS percibido sobre el posicionamiento en el mercado. En concordancia con estudios anteriores (Gonçalves et al., 2015; Hanaysha, 2018), el presente estudio expone que si 
existe relación entre las acciones de sostenibilidad y el posicionamiento en el mercado.

En segundo lugar, se observa el efecto positivo y significativo de al relación del posicionamiento en el mercado sobre el capital de marca, como ya se ha determinado en investigaciones anteriores (Fuentes-Blasco et al., 2017; Girard et al., 2017).

En tercer lugar, los resultados obtenidos permiten confirmar que el DS contribuyen significativamente al capital de marca, confirmado lo que señalan (Hampl y Loock, 2013; Lavorata, 2014).

Finalmente, se el capital de marca afecta de manera positiva y significativa a la lealtad, de este modo, cuando el consumidor de una MIPYME percibe las actividades positivas del establecimiento, genera actitudes positivas hacia la marca que incluye el compromiso a largo plazo del consumidor, es decir la lealtad.

\section{REFERENCIAS BIBLIOGRÁFICAS}

Aggeri, F., Pezet, E., Abrassart, C., \& Acquier, A. (2005). Organiser le développement durable: Expériences des entreprises pionnières et formation de règles d'action collective. Paris: Vuibert.

Álvarez, A. M. (2016). Retos De América Latina: Agenda Para El Desarrollo Sostenible Y Negociaciones Del Siglo Xxi. Problemas Del Desarrollo, 47(186), 9-30. https://doi.org/10.1016/j.rpd.2016.08.002

Andrews, M., Luo, X., Fang, Z., \& Aspara, J. (2014). Cause marketing effectiveness and the moderating role of price discounts. Journal of Marketing, 78(6), 120-142.

Arnett, D.B., Laverie, D.A. \& Meiers, A. (2003). Developing parsimonious retailer equity indexes using partial least squares analysis: a method and applications. Journal of Retailing 79(3) 161-170

Awan, M., Ho, H., \& Khan, H. (2017). Possible Effect of Merger and Acquisition on Brand Equity: A Case Study of the IT Industry in South Korea. International Journal of Business and Information. 12(1) 1-38

Bergman, M. M., Bergman, Z., \& Berger, L. (2017). An empirical exploration, typology, and definition of corporate sustainability. Sustainability, 9(5), 753.

Bermejo, R. (2014). Del desarrollo sostenible según Brundtland a la sostenibilidad como biomimesis. Hegoa, Instituto de Estudios sobre Desarrollo y Cooperación Internacional.

Bobe, C., \& Dragomir, V. D. (2010). The sustainability policy of FIVE leading european retailers. Accounting and Management Information Systems, 9(2), 268283. Retrieved from https://search.proquest.com/docview/852008903?accountid $=14777$

Cai, L., \& He, C. (2014). Corporate environmental responsibility and equity prices. Journal of Business Ethics, 125(4), 617-635.

Carmines, E.G. \& Zeller, R.A. (1975). Reliability and Validity Assessment. London: Sage Publications.

\section{Cumbres}


Ecodise, R., Issn-, S., Nacional, L., Forestales, P., Piso, T., Principal, A., ... Forestal, C. (1856). No Title, 669-673.

Fuentes-Blasco, M., Moliner-Velazquez, B., Servera-Francés, D., \& Gil-Saura, I. (2017). Role of marketing and thechnological innovation on store equity, satisfaction and Word-of-mouth in retailing. Journal of Product \& Brand Management 26(6), 650-666.

Gallarza, M. G., Ruiz-Molina, M. E, \& Gil-Saura, I. (2016). Stretching the value-satisfaction-loyalty chain by adding value dimensions and cognitive and affective satisfactions: a casual model for retailing. Management Decision, 54(4), 981-1003.

Girard, T., Trapp, P., Pinar, M., Gulsoy, T., \& Boyt, T. E. (2017). Consumer-based brand equity of a private-label brand: Measuring and examining determinants. Journal of Marketing Theory and Practice, 25(1), 39-56.

Gonçalves, M. F., Menezes, J., \& Marques, C. (2015). Grocery consumer relational perceptions in green consumption context. Tourism \& Management Studies, 11(1), 160-163.

Gulyás, E. (2011). Sustainability issues in the ethical codes of hypermarkets: A case for inefficient self-regulation. Society and Economy, 33(1), 111-129.

Hair, J. F., Hult, G.T.M., Ringle, C., \& Sarstedt, M. (2016). A primer on partial least squares structural equiation modeling (PLS-SEM). Sage Publications.

Hampl, N. \& Loock, M. (2013). Sustainable development in retailing: What is the impact on store choice?, Business Strategy and the Environment, Vol. 22(3), 202-216.

Hanaysha, J. R. (2018). Customer retention and the mediating role of perceived value in retail industry. World Journal of Entrepreneurship, Management and Sustainable Development. 14(1), 2-24.

Hooley, G.J.; Saunders, J. (1996). Posicionamiento competitivo. Sao Paulo: Makron Books

Horsky,D.; Nelson,P. (1992) New Brand Positioning and Pricing in an Oligopolistic Market Marketing Science, Vol.11 (2) 133-153

Lavorata, L. (2014). Influence of retailers' commitment to sustainable development on store image, consumer loyalty and consumer boycotts: Proposal for a model using the theory of planned behavior. Journal of Retailing and Consumer Services, 21(6), 1021-2027.

Lavorata, L., \& Mugel, O. (2017). Compréhension des mécanismes de construction des stratégies de développement durable des distributeurs indépendants: Une étude qualitative. Revue de l'organisation responsable, 12(1), 34-52.

Lombart, C., \& Louis, D. (2014). A study of the impact of Corporate Social Responsibility and price image on retailer personality and consumers' reactions-satisfaction, trust and loyalty to the retailer. Journal of Retailing and Consumer Services, 21(4), 630-642.

Minguet, P. A., \& Angels Ull Sol?s, M. (2009). La formaci?n de competencias b?sicas para el desarrollo sostenible: El papel de la universidad. Revista de Educacion, (SPEC. ISSUE), 219-237. https://doi.org/10.1017/ CBO9781107415324.004 
Nunnally, J. C., \& Bernstein, I. H. (1994). Psychometric theory. New York: McGraw-Hill.

Organización de las Naciones Unidas (ONU). (2015). Agenda 2030 A/ RES/70/1. Recuperado de http://www.un.org/sustainabledevelopment/es/ la-agenda-de-desarrollo-sostenible/

Reinartz, W., Dellaert, B., Kraft, M., Kumar, V., \& Varadarajan, R. (2011). Retailing Innovations in a Globalizing Retail Market Environment. Journal of Retailing, 87, S53-66.

Saavedra García, M. L., Moreno Uribe, H., \& Hernández Callejas, G. Y. (2008). Carecterización de las MIPYMES en Latino-América: Un estudio comparativo. Revista Internacional La Nueva Gestión Organizacional, 7(Jul-Dic), 5774. https://doi.org/ISSN: 1870205-8

Santeli, G. E. (2016). La responsabilidad social empresarial en las micro, pequeñas y medianas empresas de Ecuador. Teuken Bidikay, 08(8), 39-58. Retrieved from http://revistas.elpoli.edu.co/index.php/teu/article/view/1035

Serralvo,F.; Tadeu Furrier,M. (2005) Tipologías del posicionamiento en marcas. Un estudio conceptual en Brasil y en España. Revista Galega de Economía 14, 1-15.

Stahl, F., Heitmann, M., Lehmann, D.R. \& Neslin, S.A. (2012). The impact of brand equity on customer acquisition, retention, and profit margin. Journal of Marketing, 76(4), 44-63.

Torres-Moraga, E., Vasquez-Parraga, A.Z., \& Zamora-González, J. (2008). Customer satisfaction and loyalty: Start with the product, culminate with the brand. Journal of Consumer Marketing 25(5), 302-313

Yip, A. W., \& Bocken, N. M. (2018). Sustainable business model archetypes for the banking industry. Journal of Cleaner Production, 174, 150-169. 